\title{
Positive properties of Green's function for focal-type BVPs of singular nonlinear fractional differential equations and its application
}

Manli Jin and Yuguo Lin*

${ }^{\text {*Correspondence: }}$

yglinoa@163.com

School of Mathematics, Beihua

University, Jilin, 132013, China

\begin{abstract}
In this paper, we consider the properties of Green's function for the singular nonlinear fractional differential equation boundary value problem

$$
\begin{aligned}
& D_{0^{+}}^{\alpha} u(t)=f(t, u(t)), \quad 0<t<1, \\
& u(0)=u^{\prime}(0)=u^{\prime \prime}(1)=u^{\prime \prime \prime}(1)=0,
\end{aligned}
$$

where $3<\alpha \leq 4$ is a real number and $D_{0^{+}}^{\alpha}$ is the standard Riemann-Liouville differentiation. As an application of the properties of Green's function, we give the existence of multiple positive solutions for the above mentioned singular boundary value problems. Our tools are Leray-Schauder nonlinear alternative and Krasnoselskii's fixed-point theorem on cones.

MSC: 34A08; 34B18; 45B05
\end{abstract}

Keywords: fractional differential equation; positive solution; fractional Green's function; fixed-point theorem

\section{Introduction}

Fractional differential equations have been of great interest recently. This is due to the intensive development of the theory of fractional calculus itself as well as its applications. Apart from diverse areas of mathematics, fractional differential equations arise in rheology, dynamical processes in selfsimilar and porous structures, fluid flows, electrical networks, viscoelasticity, chemical physics, and many other branches of science. For details, see [1-6]. Now the boundary value problem for fractional differential equations attracts lots of attention. Especially, Jiang and Yuan [7] considered the nonlinear fractional differential equation Dirichlet-type boundary value problem and established the existence of positive solutions for the corresponding BVP. Xu et al. [8] dealt with the following equation:

$$
\begin{aligned}
& D_{0^{+}}^{\alpha} u(t)=f(t, u(t)), \quad 0<t<1, \\
& u(0)=u(1)=u^{\prime}(0)=u^{\prime}(1)=0 .
\end{aligned}
$$


Some properties of Green's function for the above BVP were obtained. For other related works on the fractional differential equation, see [9-15].

Motivated by the above mentioned works, we consider the properties of Green's function for

$$
\begin{aligned}
& D_{0^{+}}^{\alpha} u(t)=f(t, u(t)), \quad 0<t<1, \\
& u(0)=u^{\prime}(0)=u^{\prime \prime}(1)=u^{\prime \prime \prime}(1)=0,
\end{aligned}
$$

where $3<\alpha \leq 4$ is a real number and $D_{0^{+}}^{\alpha}$ is the standard Riemann-Liouville differentiation. As an application of Green's function, we will give the existence of multiple positive solutions for singular boundary value problems (1.1), (1.2). As far as we know, no contributions concerning BVP (1.1), (1.2) exist.

The outline of this paper is as follows. In Section 2, we derive the corresponding Green's function and some of its properties. In Section 3, by using Leray-Schauder nonlinear alternative and Krasnoselskii's fixed-point theorem in a cone, we offer criteria for the existence of positive solutions for singular BVP (1.1), (1.2).

\section{Background materials and Green's function}

For the convenience of the reader, we present here the necessary definitions from fractional calculus theory. These definitions can be found in the recent literature such as [8].

Definition 2.1 [8] The Riemann-Liouville fractional integral of order $\alpha>0$ of a function $y:(0, \infty) \rightarrow \mathbb{R}$ is given by

$$
I_{0^{+}}^{\alpha} y(t)=\frac{1}{\Gamma(\alpha)} \int_{0}^{t}(t-s)^{\alpha-1} y(s) d s
$$

provided the right-hand side is pointwise defined on $(0, \infty)$.

Definition 2.2 [8] The Riemann-Liouville fractional derivative of order $\alpha>0$ of a continuous function $y:(0, \infty) \rightarrow \mathbb{R}$ is given by

$$
D_{0^{+}}^{\alpha} y(t)=\frac{1}{\Gamma(n-\alpha)}\left(\frac{d}{d t}\right)^{n} \int_{0}^{t} \frac{y(s)}{(t-s)^{\alpha-n+1}} d s
$$

where $n=[\alpha]+1,[\alpha]$ denotes the integer part of the number $\alpha$, provided that the righthand side is pointwise defined on $(0, \infty)$.

From the definition of the Riemann-Liouville derivative, we can obtain the statement.

Lemma 2.1 [8] Let $\alpha>0$. If we assume that $u \in C(0,1) \cap L(0,1)$, then the fractional differential equation

$$
D_{0^{+}}^{\alpha} u(t)=0
$$

has $u(t)=C_{1} t^{\alpha-1}+C_{2} t^{\alpha-2}+\cdots+C_{N} t^{\alpha-N}, C_{i} \in \mathbb{R}, i=1,2, \ldots, N$, as unique solutions, where $N$ is the smallest integer greater than or equal to $\alpha$. 
Lemma 2.2 [8] Assume that $u \in C(0,1) \cap L(0,1)$ with a fractional derivative of order $\alpha>0$ that belongs to $C(0,1) \cap L(0,1)$. Then

$$
I_{0^{+}}^{\alpha} D_{0^{+}}^{\alpha} u(t)=u(t)+C_{1} t^{\alpha-1}+C_{2} t^{\alpha-2}+\cdots+C_{N} t^{\alpha-N}
$$

for some $C_{i} \in \mathbb{R}, i=1,2, \ldots, N, N$ is the smallest integer greater than or equal to $\alpha$.

In the following, we present Green's function of the fractional differential equation boundary value problem (1.1), (1.2).

Lemma 2.3 Given $h \in C[0,1]$ and $3<\alpha \leq 4$, the unique solution of

$$
\begin{aligned}
& D_{0^{+}}^{\alpha} u(t)=h(t), \quad 0<t<1, \\
& u(0)=u^{\prime}(0)=u^{\prime \prime}(1)=u^{\prime \prime \prime}(1)=0
\end{aligned}
$$

is

$$
u(t)=\int_{0}^{1} G(t, s) h(s) d s
$$

where

$$
G(t, s)= \begin{cases}\frac{(t-s)^{\alpha-1}+t^{\alpha-2}(1-s)^{\alpha-4}[(\alpha-4) t(1-s)-(\alpha-3) t+(\alpha-1) s]}{\Gamma(\alpha)}, & s \leq t, \\ \frac{t^{\alpha-2}(1-s)^{\alpha-4}[(\alpha-4) t(1-s)-(\alpha-3) t+(\alpha-1) s]}{\Gamma(\alpha)}, & t \leq s .\end{cases}
$$

Here $G(t, s)$ is called Green's function of BVP (2.1), (2.2).

Proof We may apply Lemma 2.2 to reduce (2.1) to an equivalent integral equation

$$
u(t)=I_{0^{+}}^{\alpha} h(t)+C_{1} t^{\alpha-1}+C_{2} t^{\alpha-2}+C_{3} t^{\alpha-3}+C_{4} t^{\alpha-4}
$$

for some $C_{1}, C_{2}, C_{3}, C_{4} \in \mathbb{R}$. Consequently, the general solution of (2.1) is

$$
u(t)=\frac{1}{\Gamma(\alpha)} \int_{0}^{t}(t-s)^{\alpha-1} h(s) d s+C_{1} t^{\alpha-1}+C_{2} t^{\alpha-2}+C_{3} t^{\alpha-3}+C_{4} t^{\alpha-4} .
$$

By (2.2), we get that $C_{3}=C_{4}=0$, and

$$
\begin{aligned}
& C_{1}=\frac{1}{\Gamma(\alpha)} \int_{0}^{1}\left[(\alpha-4)(1-s)^{\alpha-3}-(\alpha-3)(1-s)^{\alpha-4}\right] h(s) d s, \\
& C_{2}=\frac{\alpha-1}{\Gamma(\alpha)} \int_{0}^{1}(1-s)^{\alpha-4} \operatorname{sh}(s) d s .
\end{aligned}
$$

Therefore, the unique solution of (2.1), (2.2) is

$$
\begin{aligned}
u(t)= & \frac{1}{\Gamma(\alpha)} \int_{0}^{t}(t-s)^{\alpha-1} h(s) d s \\
& +\frac{1}{\Gamma(\alpha)} \int_{0}^{1} t^{\alpha-1}\left[(\alpha-4)(1-s)^{\alpha-3}-(\alpha-3)(1-s)^{\alpha-4}\right] h(s) d s
\end{aligned}
$$




$$
\begin{aligned}
& +\frac{\alpha-1}{\Gamma(\alpha)} \int_{0}^{1} t^{\alpha-2}(1-s)^{\alpha-4} \operatorname{sh}(s) d s \\
= & \frac{1}{\Gamma(\alpha)} \int_{0}^{t}\left\{(t-s)^{\alpha-1}+t^{\alpha-2}(1-s)^{\alpha-4}[(\alpha-4) t(1-s)-(\alpha-3) t+(\alpha-1) s]\right\} h(s) d s \\
& +\frac{1}{\Gamma(\alpha)} \int_{t}^{1} t^{\alpha-2}(1-s)^{\alpha-4}[(\alpha-4) t(1-s)-(\alpha-3) t+(\alpha-1) s] h(s) d s \\
= & \int_{0}^{1} G(t, s) h(s) d s .
\end{aligned}
$$

This completes the proof.

From the expression of $G(t, s)$, we can obtain the following properties.

Lemma 2.4 Green's function $G(t, s)$ defined by (2.3) has the following properties:

$$
\begin{aligned}
& G_{t t}(t, s) \leq 0, \quad 0 \leq s \leq t \leq 1 ; \\
& 0 \leq \Gamma(\alpha) G_{t}(t, s) \leq(\alpha-1)(\alpha-2) s^{\alpha-2}(1-s)^{\alpha-4}, \quad(t, s) \in(0,1) \times(0,1) ; \\
& (\alpha-2) s^{\alpha-2}(1-s)^{\alpha-4} t^{\alpha-2} \leq \Gamma(\alpha) G(t, s) \leq 3 s^{\alpha-2}(1-s)^{\alpha-4}, \quad(t, s) \in(0,1) \times(0,1) .
\end{aligned}
$$

\section{Proof of (2.4)}

$\Gamma(\alpha) G_{t t}(t, s)$

$$
\begin{aligned}
= & (\alpha-1)(\alpha-2)(t-s)^{\alpha-3}+(1-s)^{\alpha-4}\left[(\alpha-4)(\alpha-1)(\alpha-2) t^{\alpha-3}(1-s)\right. \\
& \left.-(\alpha-3)(\alpha-1)(\alpha-2) t^{\alpha-3}+(\alpha-1)(\alpha-2)(\alpha-3) t^{\alpha-4} s\right] \\
= & (\alpha-1)(\alpha-2)\left\{(t-s)^{\alpha-3}+(1-s)^{\alpha-4} t^{\alpha-4}[(\alpha-4) t(1-s)+(\alpha-3)(s-t)]\right\} \\
= & (\alpha-1)(\alpha-2)\left[(t-s)^{\alpha-3}-(4-\alpha)(t-t s)^{\alpha-3}-(\alpha-3)(t-s)(t-t s)^{\alpha-4}\right] \\
= & (\alpha-1)(\alpha-2)\left\{(4-\alpha)\left[(t-s)^{\alpha-3}-(t-t s)^{\alpha-3}\right]\right. \\
& \left.+(\alpha-3)(t-s)\left[(t-s)^{\alpha-4}-(t-t s)^{\alpha-4}\right]\right\} \\
= & (\alpha-1)(\alpha-2)\left[(4-\alpha)(\alpha-3) \int_{t-t s}^{t-s} x^{\alpha-4} d x\right. \\
& \left.+(\alpha-3)(\alpha-4)(t-s) \int_{t-t s}^{t-s} x^{\alpha-5} d x\right] \\
= & (\alpha-1)(\alpha-2)(\alpha-3)(4-\alpha) \int_{t-t s}^{t-s} x^{\alpha-5}(x-(t-s)) d x \\
\leq & 0, \quad 0 \leq s \leq t \leq 1,
\end{aligned}
$$

where $3<\alpha \leq 4$ and $t-t s \geq t-s$ are used. Thus, (2.4) is verified.

Proof of (2.5) By direct calculation, we get

$$
G_{t}(t, s)=\frac{\alpha-1}{\Gamma(\alpha)}\left\{\begin{array}{cc}
(t-s)^{\alpha-2}+t^{\alpha-3}(1-s)^{\alpha-4} & s \leq t, \\
\times[(\alpha-4) t(1-s)-(\alpha-3) t+(\alpha-2) s], & \\
t^{\alpha-3}(1-s)^{\alpha-4}[(\alpha-4) t(1-s)-(\alpha-3) t+(\alpha-2) s], & t \leq s .
\end{array}\right.
$$


On the one hand, for $t \geq s$, from (2.4), we can get

$$
\begin{aligned}
0 & \leq(\alpha-1)(1-s)^{\alpha-4} s^{2} \\
& =\Gamma(\alpha) G_{t}(1, s) \leq \Gamma(\alpha) G_{t}(t, s) \leq \Gamma(\alpha) G_{t}(s, s) \\
& =(\alpha-1) s^{\alpha-3}(1-s)^{\alpha-4}[(\alpha-4) s(1-s)-(\alpha-3) s+(\alpha-2) s] \\
& =(\alpha-1) s^{\alpha-2}(1-s)^{\alpha-4}[(\alpha-3)-(\alpha-4) s] \\
& \leq(\alpha-1)(\alpha-2) s^{\alpha-2}(1-s)^{\alpha-4} .
\end{aligned}
$$

On the other hand, for $t \leq s$, we have

$$
\begin{aligned}
\Gamma(\alpha) G_{t}(t, s) & =(\alpha-1) t^{\alpha-3}(1-s)^{\alpha-4}[(\alpha-4) t(1-s)-(\alpha-3) t+(\alpha-2) s] \\
& \leq(\alpha-1) s^{\alpha-3}(1-s)^{\alpha-4}(\alpha-2) s \\
& =(\alpha-1)(\alpha-2) s^{\alpha-2}(1-s)^{\alpha-4}
\end{aligned}
$$

and

$$
\begin{aligned}
\Gamma(\alpha) G_{t}(t, s) & =(\alpha-1) t^{\alpha-3}(1-s)^{\alpha-4}[(\alpha-4) t(1-s)-(\alpha-3) t+(\alpha-2) s] \\
& \geq(\alpha-1) t^{\alpha-3}(1-s)^{\alpha-4}[(\alpha-4) s-(\alpha-3) s+(\alpha-2) s] \\
& =(\alpha-1) t^{\alpha-3}(1-s)^{\alpha-4}(\alpha-3) s \geq 0 .
\end{aligned}
$$

Hence,

$$
0 \leq \Gamma(\alpha) G_{t}(t, s) \leq(\alpha-1)(\alpha-2) s^{\alpha-2}(1-s)^{\alpha-4}, \quad t \leq s .
$$

In summary, the property (2.5) holds.

Proof of (2.6) For $t \leq s$, we have

$$
\begin{aligned}
\Gamma(\alpha) G(t, s) & =t^{\alpha-2}(1-s)^{\alpha-4}[(\alpha-4) t(1-s)-(\alpha-3) t+(\alpha-1) s] \\
& \leq s^{\alpha-2}(1-s)^{\alpha-4}(\alpha-1) s \leq 3 s^{\alpha-2}(1-s)^{\alpha-4}
\end{aligned}
$$

and

$$
\begin{aligned}
\Gamma(\alpha) G(t, s) & =t^{\alpha-2}(1-s)^{\alpha-4}[(\alpha-4) t(1-s)-(\alpha-3) t+(\alpha-1) s] \\
& \geq t^{\alpha-2}(1-s)^{\alpha-4}[(\alpha-4) s-(\alpha-3) s+(\alpha-1) s] \\
& =(\alpha-2) s t^{\alpha-2}(1-s)^{\alpha-4} \\
& \geq(\alpha-2) s^{\alpha-2}(1-s)^{\alpha-4} t^{\alpha-2} .
\end{aligned}
$$

So, we have

$$
(\alpha-2) s^{\alpha-2}(1-s)^{\alpha-4} t^{\alpha-2} \leq \Gamma(\alpha) G(t, s) \leq 3 s^{\alpha-2}(1-s)^{\alpha-4}, \quad t \leq s .
$$


For $t \geq s$, we first prove that

$$
\Gamma(\alpha) G(t, s) \geq(\alpha-2) s^{\alpha-2}(1-s)^{\alpha-4} t^{\alpha-2}, \quad s \leq t .
$$

For fixed $s \in(0,1)$, let

$$
z(t)=\Gamma(\alpha) G(t, s)-(\alpha-2) s^{\alpha-2}(1-s)^{\alpha-4} t^{\alpha-2}, \quad t \in[s, 1] .
$$

Obviously, $z(s) \geq 0$ and $z^{\prime}(1) \geq 0$. Equation (2.4) together with

$$
\frac{\partial^{2}}{\partial t^{2}}\left[-(\alpha-2) s^{\alpha-2}(1-s)^{\alpha-4} t^{\alpha-2}\right]=-(\alpha-2)^{2}(\alpha-3) s^{\alpha-2}(1-s)^{\alpha-4} t^{\alpha-4} \leq 0
$$

implies that $z^{\prime \prime}(t) \leq 0, t \in[s, 1]$. Hence (2.7) holds.

By (2.5) we know that $\Gamma(\alpha) G(t, s)$ is increasing in $t$ on $[s, 1]$. Hence, for $s \leq t$,

$$
\begin{aligned}
\Gamma(\alpha) G(t, s) & \leq \Gamma(\alpha) G(1, s) \\
& =(1-s)^{\alpha-1}+(1-s)^{\alpha-4}[(\alpha-4)(1-s)-(\alpha-3)+(\alpha-1) s] \\
& =(1-s)^{\alpha-1}+(1-s)^{\alpha-4}[-1+3 s] \\
& =(1-s)^{\alpha-4}\left((1-s)^{3}-1+3 s\right) \\
& =(1-s)^{\alpha-4} s^{2}(3-s) \leq 3(1-s)^{\alpha-4} s^{\alpha-2} .
\end{aligned}
$$

In summary,

$$
(\alpha-2) s^{\alpha-2}(1-s)^{\alpha-4} t^{\alpha-2} \leq \Gamma(\alpha) G(t, s) \leq 3 s^{\alpha-2}(1-s)^{\alpha-4}, \quad s \leq t .
$$

This completes the proof of property (2.6).

As an application of properties of Green's function, we will establish the existence of positive solutions for BVP (1.1), (1.2) in Section 3, in which Leray-Schauder nonlinear alternative and Krasnoselskii's fixed-point theorem on cones are our main tools. For the convenience of the reader, we recall the two famous theorems here.

Lemma 2.5 Assume that $\Omega$ is a relative subset of a convex set $K$ in a normed space $X$. Let $A: \bar{\Omega} \rightarrow K$ be a compact map with $0 \in \Omega$. Then
(a) A has a fixed point in $\bar{\Omega}$, or
(b) there are $x \in \partial \Omega$ and $0<\lambda<1$ such that $x=\lambda A(x)$.

Lemma 2.6 Let E be a Banach space, and let $C \subset$ E be a cone in E. Assume that $\Omega_{1}, \Omega_{2}$ are open subsets of $E$ with $0 \in \Omega_{1} \subset \bar{\Omega}_{1} \subset \Omega_{2}$, and let $T: C \cap\left(\bar{\Omega}_{2} \backslash \Omega_{1}\right) \rightarrow C$ be a completely continuous operator such that either

(i) $\|T u\| \leq\|u\|, u \in C \cap \partial \Omega_{1} ;\|T u\| \geq\|u\|, u \in C \cap \partial \Omega_{2}$; or

(ii) $\|T u\| \geq\|u\|, u \in C \cap \partial \Omega_{1} ;\|T u\| \leq\|u\|, u \in C \cap \partial \Omega_{2}$.

Then $T$ has a fixed point in $C \cap\left(\bar{\Omega}_{2} \backslash \Omega_{1}\right)$. 


\section{Positive solution of a singular problem}

In this section, we establish the existence of positive solutions for singular BVP (1.1), (1.2). Throughout this section, we always assume that $f:[0,1] \times(0, \infty) \rightarrow[0, \infty)$ is continuous. Given $a \in L^{1}(0,1)$, we write $a \succ 0$ if $a \geq 0$ for $t \in[0,1]$ and it is positive in a subset of positive measure.

Let $\mathbb{C}[0,1]$ be endowed with the maximum norm, $\|u\|=\max _{0 \leq t \leq 1}|u(t)|$.

Theorem 3.1 Suppose that the following hypotheses hold:

$\left(\mathrm{H}_{1}\right)$ for each constant $L>0$, there exists a continuous function $\phi_{L} \succ 0$ such that $f(t, u) \geq$ $\phi_{L}(t)$ for all $(t, u) \in[0,1] \times(0, L]$;

$\left(\mathrm{H}_{2}\right)$ there exist continuous, nonnegative functions $g(u)$ and $h(u)$ such that

$$
0 \leq f(t, u)=g(u)+h(u) \quad \text { for all }(t, u) \in[0,1] \times(0, \infty)
$$

where $g(u)>0$ is nonincreasing and $h(u) / g(u)$ is nondecreasing in $u \in(0, \infty)$;

$\left(\mathrm{H}_{3}\right)$ there exists a constant $K_{0}>0$ such that $g(a b) \leq K_{0} g(a) g(b)$ for all $a, b>0$;

$\left(\mathrm{H}_{4}\right) \int_{0}^{1} s^{\alpha-2}(1-s)^{\alpha-4} g\left(s^{\alpha-2}\right) d s<\infty$;

$\left(\mathrm{H}_{5}\right)$ there exists a positive number $r$ such that

$$
\left\{1+\frac{h(r)}{g(r)}\right\} \frac{3 K_{0}}{\Gamma(\alpha)} g\left(\frac{(\alpha-2) r}{3}\right) \int_{0}^{1} s^{\alpha-2}(1-s)^{\alpha-4} g\left(s^{\alpha-2}\right) d s<r .
$$

Then BVP (1.1), (1.2) has at least one positive solution $u$ with $0<\|u\|<r$.

Proof Choose $n_{0} \in\{1,2, \ldots\}$ such that $n_{0}>\frac{3}{(\alpha-2) r}$. For fixed $n \in \mathbb{N}_{0}=\left\{n_{0}, n_{0}+1, \ldots\right\}$, consider the family of integral equations

$$
u(t)=\lambda \int_{0}^{1} G(t, s) f_{n}(s, u(s)) d s
$$

where $\lambda \in[0,1]$, and $f_{n}(t, u(t))=f\left(t, \max \left\{u(t), \frac{t^{\alpha-2}}{n}\right\}\right), t \in(0,1)$. We claim that any solution $u(t)$ of (3.1) for any $\lambda \in[0,1]$ must satisfy $\|u\| \neq r$. Otherwise, assume that $u(t)$ is a solution of (3.1) for some $\lambda \in[0,1]$ such that $\|u\|=r$. Note that by (2.6),

$$
\|u\| \leq \frac{3}{\Gamma(\alpha)} \lambda \int_{0}^{1} s^{\alpha-2}(1-s)^{\alpha-4} f_{n}(s, u(s)) d s
$$

Hence, for all $t \in[0,1]$, by (2.6) again, we have

$$
\begin{aligned}
u(t) & \geq \frac{(\alpha-2) t^{\alpha-2}}{\Gamma(\alpha)} \lambda \int_{0}^{1} s^{\alpha-2}(1-s)^{\alpha-4} f_{n}(s, u(s)) d s \\
& \geq \frac{(\alpha-2) t^{\alpha-2}}{3}\|u\|=\frac{(\alpha-2) r t^{\alpha-2}}{3}
\end{aligned}
$$


Thus, it follows from the choice of $n_{0},(3.2),(2.6),\left(\mathrm{H}_{2}\right)$ and $\left(\mathrm{H}_{3}\right)$ that for all $t \in[0,1]$,

$$
\begin{aligned}
u(t) & =\lambda \int_{0}^{1} G(t, s) f_{n}(s, u(s)) d s \\
& =\lambda \int_{0}^{1} G(t, s) f(s, u(s)) d s \\
& \leq \frac{3}{\Gamma(\alpha)} \int_{0}^{1} s^{\alpha-2}(1-s)^{\alpha-4} g(u(s))\left\{1+\frac{h(u(s))}{g(u(s))}\right\} d s \\
& \leq\left\{1+\frac{h(r)}{g(r)}\right\} \frac{3}{\Gamma(\alpha)} \int_{0}^{1} s^{\alpha-2}(1-s)^{\alpha-4} K_{0} g\left(\frac{(\alpha-2) r}{3}\right) g\left(s^{\alpha-2}\right) d s \\
& =\left\{1+\frac{h(r)}{g(r)}\right\} \frac{3 K_{0}}{\Gamma(\alpha)} g\left(\frac{(\alpha-2) r}{3}\right) \int_{0}^{1} s^{\alpha-2}(1-s)^{\alpha-4} g\left(s^{\alpha-2}\right) d s .
\end{aligned}
$$

Therefore,

$$
r=\|u\| \leq\left\{1+\frac{h(r)}{g(r)}\right\} \frac{3 K_{0}}{\Gamma(\alpha)} g\left(\frac{(\alpha-2) r}{3}\right) \int_{0}^{1} s^{\alpha-2}(1-s)^{\alpha-4} g\left(s^{\alpha-2}\right) d s,
$$

which contradicts $\left(\mathrm{H}_{5}\right)$ and the claim is proved.

It is easy to check from (2.5), (2.6) and $\left(\mathrm{H}_{4}\right)$ that the operator

$$
S_{n}(t)=\int_{0}^{1} G(t, s) f_{n}(s, u(s)) d s
$$

is completely continuous, where $n \in \mathbb{N}_{0}$. We omit the details here.

Now Lemma 2.5 guarantees that the integral equation $u(t)=S_{n}(t)$ has a solution, denoted by $u_{n}$, in $\bar{B}_{r}=\{u \in C[0,1]:\|u\| \leq r\}$.

From $\left(\mathrm{H}_{1}\right)$, we know that there exists a function $\phi_{r} \succ 0$ such that $f(t, u) \geq \phi_{r}(t)$ for all $(t, u) \in[0,1] \times(0, r]$,

$$
\begin{aligned}
u_{n}(t) & =\int_{0}^{1} G(t, s) f_{n}(s, u(s)) d s \\
& \geq \frac{(\alpha-2) t^{\alpha-2}}{\Gamma(\alpha)} \int_{0}^{1} s^{\alpha-2}(1-s)^{\alpha-4} \phi_{r}(s) d s=\delta t^{\alpha-2}
\end{aligned}
$$

where $\delta:=\frac{(\alpha-2)}{\Gamma(\alpha)} \int_{0}^{1} s^{\alpha-2}(1-s)^{\alpha-4} \phi_{r}(s) d s>0$.

By using (2.5), (3.5) and a similar calculation as in (3.3), we have that for all $n \in \mathbb{N}_{0}$,

$$
\left\|u_{n}\right\| \leq r
$$

and

$$
\left\|u_{n}^{\prime}\right\| \leq\left\{1+\frac{h(r)}{g(r)}\right\} \frac{(\alpha-1)(\alpha-2) K_{0}}{\Gamma(\alpha)} g(\delta) \int_{0}^{1} s^{\alpha-2}(1-s)^{\alpha-4} g\left(s^{\alpha-2}\right) d s<\infty .
$$

The Arzela-Ascoli theorem guarantees that $\left\{u_{n}\right\}_{n \in \mathbb{N}_{0}}$ has a subsequence $\left\{u_{n_{k}}\right\}_{k \in \mathbb{N}}$ converging uniformly on $[0,1]$ to a function $u \in C[0,1]$. By the Lebesgue dominated convergence 
theorem, we have that

$$
u(t)=\int_{0}^{1} G(t, s) f(s, u(s)) d s
$$

where (3.5) is used. Therefore, $u(t)$ is a positive solution of BVP (1.1), (1.2).

Theorem 3.2 Suppose that $\left(\mathrm{H}_{2}\right),\left(\mathrm{H}_{3}\right),\left(\mathrm{H}_{4}\right)$ and $\left(\mathrm{H}_{5}\right)$ are satisfied. Furthermore, assume that $\left(\mathrm{H}_{6}\right)$ there exists a positive number $R>r$ such that the following inequality holds:

$$
\frac{\alpha-2}{\Gamma(\alpha)} g(R) \int_{0}^{1} s^{\alpha-2}(1-s)^{\alpha-4}\left\{1+\frac{h\left(\frac{(\alpha-2) s^{\alpha-2} R}{3}\right)}{g\left(\frac{(\alpha-2) s^{\alpha-2} R}{3}\right)}\right\} d s \geq R
$$

Then BVP (1.1), (1.2) has another solution $\tilde{u}$ with $r<\|\tilde{u}\|<R$.

Proof To show the existence of $\tilde{u}$, we will use Lemma 2.6. Define

$$
K=\left\{u \in C[0,1]: u(t) \geq \frac{(\alpha-2) t^{\alpha-2}}{3}\|u\| \text { for } t \in[0,1]\right\} .
$$

It is obvious that $K$ is a cone on $C[0,1]$. Let

$$
\begin{aligned}
& \Omega_{1}=\{u \in C[0,1]:\|u\|<r\}, \\
& \Omega_{2}=\{u \in C[0,1]:\|u\|<R\} .
\end{aligned}
$$

Next, the operator $T: K \cap\left(\bar{\Omega}_{2} \backslash \Omega_{1}\right) \rightarrow C[0,1]$ is defined by

$$
(T u)(t)=\int_{0}^{1} G(t, s) f(s, u(s)) d s .
$$

It is easy to check that the operator $T$ maps $K \cap\left(\bar{\Omega}_{2} \backslash \Omega_{1}\right)$ into $K$. In fact, for any $u \in$ $K \cap\left(\bar{\Omega}_{2} \backslash \Omega_{1}\right)$, we have from (2.6) that for $t \in[0,1]$,

$$
\|T u\| \leq \frac{3}{\Gamma(\alpha)} \int_{0}^{1} s^{\alpha-2}(1-s)^{\alpha-4} f(s, u(s)) d s
$$

and

$$
(T u)(t) \geq \frac{(\alpha-2) t^{\alpha-2}}{\Gamma(\alpha)} \int_{0}^{1} s^{\alpha-2}(1-s)^{\alpha-4} f(s, u(s)) d s .
$$

This implies that $(T u)(t) \geq \frac{(\alpha-2) t^{\alpha-2}}{3}\|T u\|$, that is, $T: K \cap\left(\bar{\Omega}_{2} \backslash \Omega_{1}\right) \rightarrow K$. In addition, by a similar argument as in Theorem 3.1, it is not difficult to prove that the operator $T$ : $K \cap\left(\bar{\Omega}_{2} \backslash \Omega_{1}\right) \rightarrow K$ is completely continuous. Now we prove that

$$
\|T u\| \leq\|u\|, \quad \forall u \in K \cap \partial \Omega_{1} .
$$


For any $u \in K \cap \partial \Omega_{1}$, from (2.6), $\left(\mathrm{H}_{2}\right),\left(\mathrm{H}_{3}\right),(3.6)$ and $\left(\mathrm{H}_{5}\right)$, we have that for $t \in[0,1]$,

$$
\begin{aligned}
T u(t) & =\int_{0}^{1} G(t, s) f(s, u(s)) d s \\
& \leq \frac{3}{\Gamma(\alpha)} \int_{0}^{1} s^{\alpha-2}(1-s)^{\alpha-4} g(u(s))\left\{1+\frac{h(u(s))}{g(u(s))}\right\} d s \\
& \leq\left\{1+\frac{h(r)}{g(r)}\right\} \frac{3}{\Gamma(\alpha)} \int_{0}^{1} s^{\alpha-2}(1-s)^{\alpha-4} K_{0} g\left(\frac{(\alpha-2) r}{3}\right) g\left(s^{\alpha-2}\right) d s \\
& =\left\{1+\frac{h(r)}{g(r)}\right\} \frac{3 K_{0}}{\Gamma(\alpha)} g\left(\frac{(\alpha-2) r}{3}\right) \int_{0}^{1} s^{\alpha-2}(1-s)^{\alpha-4} g\left(s^{\alpha-2}\right) d s \\
& <r=\|u\| .
\end{aligned}
$$

Therefore, (3.7) holds. Next, we will prove that

$$
\|T u\| \geq\|u\|, \quad \forall u \in K \cap \partial \Omega_{2} .
$$

For any $u \in K \cap \partial \Omega_{2}$, from (2.6), $\left(\mathrm{H}_{2}\right),\left(\mathrm{H}_{3}\right),(3.6)$ and $\left(\mathrm{H}_{6}\right)$, we have that

$$
\begin{aligned}
(T u)(1) & =\int_{0}^{1} G(1, s) f(s, u(s)) d s \\
& \geq \frac{\alpha-2}{\Gamma(\alpha)} \int_{0}^{1} s^{\alpha-2}(1-s)^{\alpha-4} g(u(s))\left\{1+\frac{h(u(s))}{g(u(s))}\right\} d s \\
& \geq \frac{\alpha-2}{\Gamma(\alpha)} g(R) \int_{0}^{1} s^{\alpha-2}(1-s)^{\alpha-4}\left\{1+\frac{h\left(\frac{(\alpha-2) s^{\alpha-2} R}{3}\right)}{g\left(\frac{(\alpha-2) s^{\alpha-2} R}{3}\right)}\right\} d s \\
& \geq R=\|u\| .
\end{aligned}
$$

This implies that (3.8) holds.

It follows from Lemma 2.6 that the operator $T$ has a fixed point $\tilde{u} \in K \cap\left(\bar{\Omega}_{2} \backslash \Omega_{1}\right)$. Clearly, this fixed point $\tilde{u}$ is a positive solution of BVP (1.1), (1.2) satisfying $r<\|\tilde{u}\| \leq R$.

Example 3.1 Consider the boundary value problem

$$
\begin{aligned}
& D_{0^{+}}^{\alpha} u(t)=u^{-a}(t)+\mu u^{b}(t), \quad t \in(0,1), \\
& u(0)=u^{\prime}(0)=u^{\prime \prime}(1)=u^{\prime \prime \prime}(1)=0,
\end{aligned}
$$

where $a>0, b \geq 0$ and $\mu>0$ is a given parameter.

Corollary 3.1 Assume that $0<a<\frac{\alpha-1}{\alpha-2}, b \geq 0$.

(i) If $b<1$, then (3.9) has at least one nonnegative solution for each $\mu>0$.

(ii) If $b \geq 1$, then (3.9) has at least one nonnegative solution for each $0<\mu<\mu_{1}$, where $\mu_{1}$ is some positive constant.

(iii) If $b>1$, then (3.9) has at least two nonnegative solutions for each $0<\mu<\mu_{1}$. 
Proof We will apply Theorems 3.1 and 3.2 to obtain our desired results. Note that $\left(\mathrm{H}_{1}\right)$ holds with $\phi_{L}(t)=L^{-a}$. Let

$$
g(u)=u^{-a}, \quad h(u)=\mu u^{b}, \quad K_{0}=1 .
$$

Then $\left(\mathrm{H}_{2}\right)$ and $\left(\mathrm{H}_{3}\right)$ are satisfied. Since $0<a<\frac{\alpha-1}{\alpha-2},\left(\mathrm{H}_{4}\right)$ is also satisfied. Now for $\left(\mathrm{H}_{5}\right)$ to be satisfied, we need

$$
\mu<\frac{A r^{1+a}-1}{r^{a+b}}
$$

for some $r>0$, where

$$
A=\left[\frac{3^{1+a}(\alpha-2)^{-a}}{\Gamma(\alpha)} \int_{0}^{1} s^{(\alpha-2)(1-a)}(1-s)^{\alpha-4} d s\right]^{-1} .
$$

Therefore, (3.9) has at least one nonnegative solution for

$$
0<\mu<\mu_{1}:=\sup _{r>0} \frac{A r^{1+a}-1}{r^{a+b}} .
$$

Note that if $b<1, \mu_{1}=\infty$. If $b>1$, set

$$
l(r):=\frac{A r^{1+a}-1}{r^{a+b}}
$$

The function $l(r)$ possesses a maximum at

$$
r_{0}:=\left(\frac{a+b}{(b-1) A}\right)^{\frac{1}{a+1}}>\left(\frac{1}{A}\right)^{\frac{1}{a+1}}
$$

then $\mu_{1}=l\left(r_{0}\right)>0$. We have the desired results (i) and (ii). If $b>1$, condition $\left(\mathrm{H}_{6}\right)$ becomes

$$
\mu \geq \frac{R^{1+a}-B}{C R^{a+b}}
$$

for some $R>0$, where

$$
\begin{aligned}
& B=\frac{\alpha-2}{\Gamma(\alpha)} \int_{0}^{1} s^{\alpha-2}(1-s)^{\alpha-4} d s, \\
& C=\frac{(\alpha-2)^{a+b+1}}{3^{a+b} \Gamma(\alpha)} \int_{0}^{1} s^{(\alpha-2)(a+b+1)}(1-s)^{\alpha-4} d s .
\end{aligned}
$$

Since $b>1$, the right-hand side of (3.10) tends to 0 as $R \rightarrow+\infty$. Thus, for any given $0<$ $\mu<\mu_{1}$, it is always possible to find an $R \gg r$ such that (3.10) is satisfied. Therefore, (3.9) has another nonnegative solution $\tilde{u}$. This implies that (iii) holds. 
Authors' contributions

All authors contributed equally in this article. They read and approved the final manuscript.

\section{Acknowledgements}

The author Yuguo Lin would like to express his gratitude to Professor Daqing Jiang for his careful direction. This work was partially supported by NSFC of China (No. 11201008).

Received: 22 May 2013 Accepted: 22 May 2013 Published: 6 June 2013

\section{References}

1. Kilbas, AA, Srivastava, HM, Trujillo, JJ: Theory and Applications of Fractional Differential Equations. North-Holland Mathematics Studies, vol. 204. Elsevier, Amsterdam (2006)

2. Oldham, KB, Spanier, J: The Fractional Calculus. Academic Press, New York (1974)

3. Nonnenmacher, TF, Metzler, R: On the Riemann-Liouville fractional calculus and some recent applications. Fractals 3 , 557-566 (1995)

4. Tatom, FB: The relationship between fractional calculus and fractals. Fractals 3, 217-229 (1995)

5. Podlubny, I: Fractional Differential Equations. Mathematics in Science and Engineering, vol. 198. Academic Press, New York (1999)

6. Samko, SG, Kilbas, AA, Marichev, Ol: Fractional Integral and Derivatives: Theory and Applications. Gordon \& Breach, Yverdon (1993)

7. Jiang, D, Yuan, C: The positive properties of the Green function for Dirichlet-type boundary value problems of nonlinear fractional differential equations and its application. Nonlinear Anal. 72, 710-719 (2010)

8. Xu, X, Jiang, D, Yuan, C: Multiple positive solutions for the boundary value problem of a nonlinear fractiona differential equation. Nonlinear Anal. 71, 4676-4688 (2009)

9. Babakhani, A, Gejji, VD: Existence of positive solutions of nonlinear fractional differential equations. J. Math. Anal. Appl. 278, 434-442 (2003)

10. Delbosco, D, Rodino, L: Existence and uniqueness for a nonlinear fractional differential equation. J. Math. Anal. Appl. 204, 609-625 (1996)

11. Zhang, S: The existence of a positive solution for a nonlinear fractional differential equation. J. Math. Anal. Appl. 252, 804-812 (2000)

12. Zhang, S: Existence of positive solution for some class of nonlinear fractional differential equations. J. Math. Anal. Appl. 278, 136-148 (2003)

13. Nakhushev, AM: The Sturm-Liouville problem for a second order ordinary differential equation with fractional derivatives in the lower terms. Dokl. Akad. Nauk SSSR 234, 308-311 (1977)

14. Bai, Z, Lü, H: Positive solutions for boundary value problem of nonlinear fractional differential equation. J. Math. Anal. Appl. 311, 495-505 (2005)

15. Benchohra, M, Graef, JR, Hamani, S: Existence results for boundary value problems with nonlinear fractional differential equations. Appl. Anal. 87(7), 851-863 (2008)

doi:10.1186/1687-1847-2013-159

Cite this article as: Jin and Lin: Positive properties of Green's function for focal-type BVPs of singular nonlinear fractional differential equations and its application. Advances in Difference Equations 2013 2013:159.

\section{Submit your manuscript to a SpringerOpen ${ }^{\circ}$ journal and benefit from:}

- Convenient online submission

Rigorous peer review

- Immediate publication on acceptance

- Open access: articles freely available online

- High visibility within the field

- Retaining the copyright to your article 